\title{
\begin{tabular}{l|l} 
Mitraries & DSpace@MIT
\end{tabular}
}

\author{
MIT Open Access Articles
}

\section{A Magnetic Retrieval System for Stents in the Pancreaticobiliary Tree}

The MIT Faculty has made this article openly available. Please share how this access benefits you. Your story matters.

Citation: Cantillon-Murphy, P. et al. “A Magnetic Retrieval System for Stents in the Pancreaticobiliary Tree." Biomedical Engineering, IEEE Transactions on 57.8 (2010): 2018-2025. (C) 2011 IEEE.

As Published: http://dx.doi.org/10.1109/tbme.2010.2045653

Publisher: Institute of Electrical and Electronics Engineers

Persistent URL: http://hdl.handle.net/1721.1/67500

Version: Final published version: final published article, as it appeared in a journal, conference proceedings, or other formally published context

Terms of Use: Article is made available in accordance with the publisher's policy and may be subject to US copyright law. Please refer to the publisher's site for terms of use. 


\title{
A Magnetic Retrieval System for Stents in the Pancreaticobiliary Tree
}

\author{
Pádraig Cantillon-Murphy*, Member, IEEE, Marvin Ryou, Sohail N. Shaikh, Dan Azagury, Michele Ryan, \\ Christopher C. Thompson, and Jeffrey H. Lang, Fellow, IEEE
}

\begin{abstract}
Clinical endoscopic intervention of the pancreaticobiliary tree [endoscopic retrograde cholangiopancreatography (ERCP)] often concludes with the insertion of a temporary plastic stent to reduce the risk of post-ERCP complications by promoting continued flow of bile and pancreatic fluids. This stent is later removed once the patient has fully recovered, but today this necessitates a second endoscopic intervention. The final goal of this work is to obviate the second intervention. This is to be achieved by adding a magnetic ring to the stent such that the stent is removed using a hand-held magnet, held in a suitable position $e x$ vivo. This paper details the design, optimization, and both $e x$ vivo and in vivo testing of the magnetized stent and hand-held magnet, which has been accomplished to date. The optimized design for the hand-held magnet and the modified stent with a magnetic attachment performs in line with simulated expectations, and successful retrieval is achieved in the porcine $e x$ vivo setting at $9-10 \mathrm{~cm}$ separation. This is comparable to the mean target capture distance of $10 \mathrm{~cm}$ between the entry point to the biliary system and the closest cutaneous surface, determined from random review of clinical fluoroscopies in ten human patients. Subsequently, the system was successfully tested in vivo in the acute porcine model, where retrieval at an estimated separation of 5-6 cm was captured on endoscopic video. These initial results indicate that the system may represent a promising approach for the elimination of a second endoscopic procedures following placement of pancreatic and biliary stents.
\end{abstract}

Index Terms-Biliary stent, magnetic retrieval, magnetic stent, pancreatic stent.

\section{INTRODUCTION}

W HEN the biliary or pancreatic ducts become occluded, the placement of a temporary, removable plastic tube (stent) facilitates bypass of the occlusion [1], [2]. Stents are also used for prophylaxis of post-endoscopic retrograde cholangiopancreatography (ERCP) complications following aggres-

Manuscript received December 10, 2009; revised January 20, 2010; accepted March 5, 2010. Date of publication May 17, 2010; date of current version July 14, 2010. Asterisk indicates corresponding author.

${ }^{*} \mathrm{P}$. Cantillon-Murphy is with the Department of Electrical Engineering and Computer Science, Massachusetts Institute of Technology, Cambridge, MA 02139 USA. He is also with the Division of Gastroenterology, Brigham and Women's Hospital, Boston, MA 02115 USA (e-mail: padraig@mit.edu).

M. Ryou, D. Azagury, M. Ryan, and C.C. Thompson are with the Division of Gastroenterology, Brigham and Women's Hospital, Boston, MA 02115 USA (e-mail: mryou@ partners.org; dazagury@ partners.org; mryan15@ partners.org; ccthompson@partners.org).

S. N. Shaikh is with the University Medical Center, University of Arizona, Tucson, AZ 85724 USA (e-mail: sohail.n.shaikh@gmail.com).

J. H. Lang is with the Department of Electrical Engineering and Computer Science, Massachusetts Institute of Technology, Cambridge, MA 02139 USA (e-mail: lang@mit.edu).

Digital Object Identifier 10.1109/TBME.2010.2045653 sive endoscopic manipulation of the pancreaticobiliary tree [3]. Typically, biliary and pancreatic stents are $4-6 \mathrm{~cm}$ in length and $1.6 \mathrm{~mm}$ (conventionally referred to as " 5 French" (5 Fr), where the difference is a factor of $\pi$ ) in diameter. Conventional stents for this purpose are commonly made of polyethylene or Teflon and often include barbs, flaps, or flanges at either one or both ends of the stent. These barbs are designed to prevent migration of the stent further up the duct (thus complicating retrieval) or premature escape from the duct. However, barbs are often removed by physicians prior to placement to accelerate the stent's expulsion from the biliary tree.

Stents have a multitude of designs either to reduce migration and/or to assist in drainage. Stents have also been formed in expandable formations to assist with drainage capabilities [4]. Both biliary and pancreatic stents are usually designed to be inserted over an endoscopic guidewire and are pushed into position with the aid of a catheter. The stent is generally advanced endoscopically until about $1 \mathrm{~cm}$ of the stent extrudes from pancreaticobiliary system into the small intestine after placement. The placement procedure ends by the withdrawal of the guiding catheter, followed by the removal of the guidewire itself. Later removal of the plastic stent (typically 2 to 3 weeks after placement) requires at least one endoscopic procedure, and can involve surgical intervention in cases where a stent migrates and lodges in the pancreatic or biliary ducts. The attractive magnetic retrieval proposed here is equally applicable to pancreatic or biliary ductal stents.

The use of magnetic retrieval for in vivo bodies is not a new concept. The magnetic retrieval of foreign bodies in the esophagus, stomach, and duodenum was first proposed by Equen et al. [5] in 1957. More recently, foreign body retrieval in the gastrointestinal tract has been augmented by fluoroscopic imaging [6], [7]. Magnetized or magnetic stents have also been widely employed in coronary procedures, where stenting is used extensively in the carotid arteries [8]-[12]. Magnetic guidance has also been proposed for nasoenteral feeding tube placement [13], [14], where an external, hand-held magnet guides the feeding tube through the esophageal tract to the subject's duodenum. Various magnetic stents and implants have also been proposed to trap magnetic drug-carrying nanoparticles in the human vasculature [15]-[20]. Additionally, navigation of larger ferromagnetic devices has been proposed [21], [22] using magnetic resonance imaging in the carotid arteries.

This study examines the design and both ex vivo and in vivo testing of a modified stent suitable for the biliary and pancreatic ducts, which can be located and removed without endoscopic or surgical intervention (i.e., without using forceps or snares). 
(a)

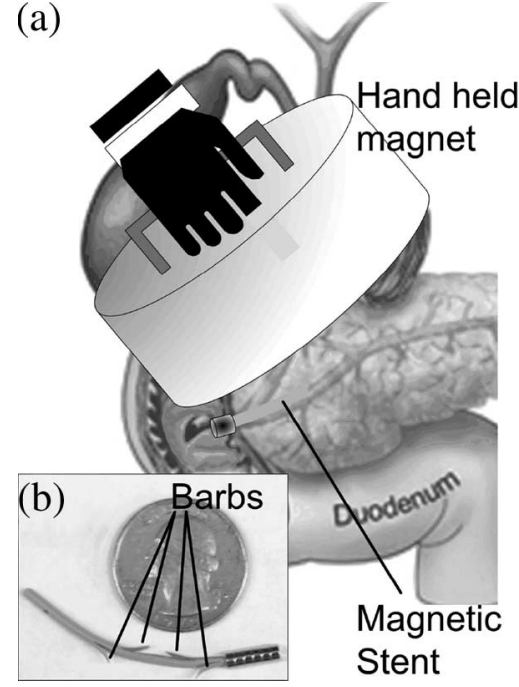

Fig. 1. Proposed system, shown in (a), uses the force of magnetic attractive between an external, hand-held magnet and a modified biliary or pancreatic stent to remove the stent from the biliary tree without the need for an endoscopic intervention. Modified stent, shown in (b), is comprised of a standard pancreatic stent attached to a cylindrical magnetic ring. For successful in vivo retrieval, it was found necessary to remove all barbs.

Instead, retrieval is by means of a ring of permanent magnetic material [neodymium-iron-boron $(\mathrm{NdFeB})]$, which is fixed to the stent's distal end and a large hand-held magnet. The magnetic ring is designed such that there is no occlusion of the stent associated with its magnetic properties, while the ring's outer diameter (OD) facilitates endoscopic deployment. The proposed system is illustrated in Fig. 1. Rather than an ad hoc testing of various magnets, a careful analytical study was undertaken to optimize the design of the hand-held magnet. This is because, in order for the system to be ultimately successfully adopted into the clinical environment, the weight of the hand-held magnet should be an absolute minimum, thus, allowing ease of use, conceivably by non-clinicians. The stent retrieval mechanism proposed and investigated in this study somewhat resembles the nanoenteral feeding tubes approach of Gabriel et al. [13], [14]. Due to the confined space of the biliary and pancreatic duct, forceps and snares do not tend to be efficient or effective in the removal of the stents. It is proposed that the modified system presented here will ultimately obviate the need for a second endoscopic intervention, thus, decreasing hospital costs, reducing patient exposure to sedation and/or anesthesia, and decreasing patient recovery time.

\section{Methods AND Results}

The hand-held permanent magnet was designed within weight constraints, and its design was optimized for maximum attractive force of retrieval at $10 \mathrm{~cm}$ separation between the hand-held magnet and the magnetic stent attachment. The OD and inner diameter (ID) of the magnetic ring were determined by the ID of the standard gastroscope's instrument channel $(\sim 5 \mathrm{~mm})$ and the current diameter of biliary stents (which can vary widely), respectively. The attractive force between the optimized handheld magnet and the magnetic stent attachment was then simu- lated and tested experimentally. The design was investigated $e x$ vivo in the porcine cadaver to demonstrate capture as a function of separation distance from the hand-held magnet after manual stent placement in the porcine cadaveric hepatopancreatic ampulla (i.e., the duct leading to the pancreaticobiliary tree). Finally, the magnetic stent was endoscopically deployed in vivo in an acute porcine model and retrieved by means of the external hand-held magnet. Magnetic retrieval was recorded using endoscopic video.

\section{A. Hand-Held Magnet and Stent Design}

The dimensions of the hand-held magnet (ratio of height to diameter) was optimized for a fixed weight. Optimization was achieved by means of numerical simulation using MATLAB (Mathworks, Inc., Natick, MA). The hand-held magnet must be sufficiently lightweight to allow easy maneuvering in the vicinity of the patient once stent retrieval is required. This magnet will ultimately be maneuvered in skin contact with the patient's stomach or side to retrieve the magnetic stent. Hand-held magnets of $2.27,3.18$, and $4.54 \mathrm{~kg}(5,7$, and $10 \mathrm{lbs})$ were investigated by means of numerical simulation. To maximize magnetic attractive forces while minimizing sharp edges and corners, a cylindrical, axially magnetized $\mathrm{N}-42$ grade NdFeB hand-held magnet was proposed and simulated, where $\mathrm{N}-42 \mathrm{NdFeB}$ is typically supplied with a remnant magnetization of $\mu_{0} M_{r}$ near $1.3 \mathrm{~T}$ (KJ Magnetics, Jamison, PA) and $\mu_{0}=4 \pi \times 10^{-7}$ is the magnetic permeability of free space. A range of hand-held magnet diameters was investigated up to $40 \mathrm{~cm}$, where the corresponding magnet depth was determined by the fixed magnet weight; $\mathrm{N}-42$ grade $\mathrm{NdFeB}$ has a density of $8.2 \mathrm{~g} / \mathrm{cm}^{3}$. Attractive force to a magnetic ring was evaluated at $10 \mathrm{~cm}$ separation. A separation of $10 \mathrm{~cm}$ was found to be typical under fluoroscopic review of the distance from the hepatopancreatic ampulla to the closest cutaneous site in ten human patients at the Endoscopy Center, Brigham and Women's Hospital, Boston.

The magnetic attachment to the stent consists of an axially magnetized cylindrical ring with an ID and OD, as shown in Fig. 1(b). The ID is chosen such that the attachment can be slipped over and adhered to the OD of the biliary or pancreatic stents $(\sim 0.625 \mathrm{~mm})$. Stents are categorized by length (from the distal end to the last barb) and external diameter so that a 5-3 stent has a $5 \mathrm{Fr}(0.16 \mathrm{~cm})$ diameter and $3 \mathrm{~cm}$ length to the last barb. The total length is approximately $4 \mathrm{~cm}$. For the following simulated and experimental results, the ring's OD is $3.175 \mathrm{~mm}\left(1 / 8^{\prime \prime}\right)$ and the ID is $1.5875 \mathrm{~mm}\left(1 / 16^{\prime \prime}\right)$ in each case, while the length is $6.35 \mathrm{~mm}\left(1 / 4^{\prime \prime}\right)$, unless otherwise stated. This OD was chosen to allow the magnetic stent to be deployed through the instrument channel of a standard endscopic gastroscope.

The attractive force was simulated using a magnetic charge model [23], where the pole faces (North/South) of both the hand-held cylindrical and stent ring magnet were quantized to represent surfaces with discrete magnetic facet charges. Each facet has an area of $d A_{1}$ for the hand-held magnet and $d A_{2}$ for the stent ring magnet, and the associated facet surface charges 
$q_{m, 1}$ and $q_{m, 2}$ are given by (1)

$$
\begin{aligned}
& q_{m, 1}=\mu_{0} M_{r} d A_{1} \\
& q_{m, 2}=\mu_{0} M_{r} d A_{2} .
\end{aligned}
$$

The magnetic force between two magnetic facets charge is then given by (2), where the indexes 1 and 2 represent the handheld magnet (index $=1$ ) and the magnetic ring (to be attached to the stent) (index $=2$ ), and $r_{12}$ is the interfacet distance between faces

$$
f_{m}=\frac{1}{4 \pi \mu_{0}} \frac{q_{m, 1} q_{m, 2}}{\left|r_{12}\right|^{2}} .
$$

The total force $F_{m}$ between the hand-held magnet and the magnetic stent attachment is due to the two pole faces (north denoted by + , and south denoted by - ) of each magnet. The total force is then the sum of the integrands on the pole faces of each magnet with areas $A_{1}^{+}$and $A_{1}^{-}$for the north and south faces, respectively, of the hand-held magnet, and areas $A_{2}^{+}$and $A_{2}^{-}$for the north and south faces, respectively, of the magnetic ring. The interfacet displacement is now denoted $r_{1+2}$, where the signs indicate which face is being considered (i.e., ${ }^{+}$for north, ${ }^{-}$for south)

$$
\begin{aligned}
F_{m}= & \frac{1}{4 \pi \mu_{0}}\left(\int_{A_{1}^{+}} \int_{A_{2}^{+}} \frac{q_{m, 1^{+}} q_{m, 2^{+}}}{\left|r_{1^{+} 2^{+}}\right|^{2}} d A_{2}^{+} d A_{1}^{+}\right. \\
& +\int_{A_{1}^{-}} \int_{A_{2}^{+}} \frac{q_{m, 1^{-}} q_{m, 2^{+}}}{\left|r_{1^{-} 2^{+}}\right|^{2}} d A_{2}^{+} d A_{1}^{-} \\
& +\int_{A_{1}^{+}} \int_{A_{2}^{-}} \frac{q_{m, 1^{+}} q_{m, 2^{-}}}{\left|r_{1^{+} 2^{-}}\right|^{2}} d A_{2}^{-} d A_{1}^{+} \\
& \left.+\int_{A_{1}^{-}} \int_{A_{2}^{-}} \frac{q_{m, 1^{-}} q_{m, 2^{-}}}{\left|r_{1^{-} 2^{-}}\right|^{2}} d A_{2}^{-} d A_{1}^{-}\right) .
\end{aligned}
$$

For this study, symmetry assures that $A_{1}^{+}=A_{1}^{-}=A_{1}$ and $A_{2}^{+}=A_{2}^{-}=A_{2}$, while for the facet areas $d A_{1}^{+}=d A_{1}^{-}=$ $d A_{1}$ and $d A_{2}^{+}=d A_{2}^{-}=d A_{2}$. For the facet charges, $q_{m, 1^{+}}=$ $-q_{m, 1^{-}}=q_{m, 1}$ and $q_{m, 2^{+}}=-q_{m, 2^{-}}=q_{m, 2}$; Therefore, simplification of (3) is possible as given in (4). The simulated problem is shown in Fig. 2

$$
\begin{aligned}
F_{m}= & \frac{1}{4 \pi \mu_{0}}\left(\int_{A_{1}} \int_{A_{2}} \frac{q_{m, 1} q_{m, 2}}{\left|r_{1^{+} 2^{+}}\right|^{2}} d A_{2} d A_{1}\right. \\
& +\int_{A_{1}} \int_{A_{2}} \frac{\left(-q_{m, 1}\right) q_{m, 2}}{\left|r_{1-2^{+}}\right|^{2}} d A_{2} d A_{1} \\
& +\int_{A_{1}} \int_{A_{2}} \frac{q_{m, 1}\left(-q_{m, 2}\right)}{\left|r_{1^{+} 2^{-}}\right|^{2}} d A_{2} d A_{1} \\
& \left.+\int_{A_{1}} \int_{A_{2}} \frac{\left(-q_{m, 1}\right)\left(-q_{m, 2}\right)}{\left|r_{1^{-} 2^{-}}\right|^{2}} d A_{2} d A_{1}\right) .
\end{aligned}
$$

Integration in the continuous domain corresponds to numerical summation in the discrete domain, as indicted in (5), where $i_{A_{1}}$ and $i_{A_{2}}$ are the numerical iterators for the face areas

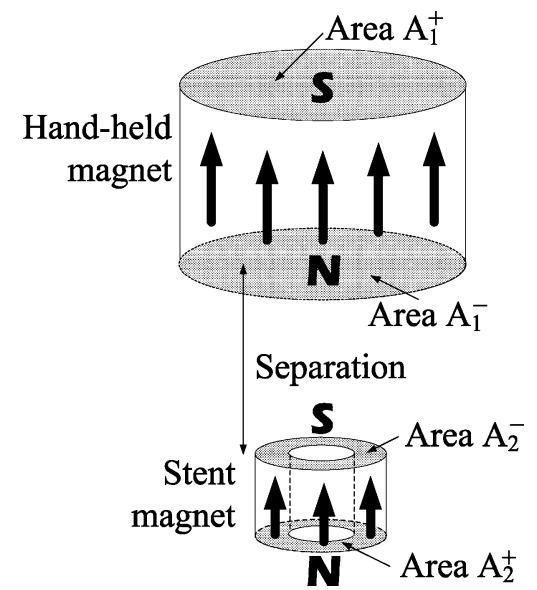

Fig. 2. Numerical simulation evaluated the force of attraction between a larger cylindrical magnet (the hand-held magnet) and a magnetic ring (for attachment to the stent) at various separations.

$A_{1}$ and $A_{2}$

$$
\begin{aligned}
F_{m}= & \frac{1}{4 \pi \mu_{0}}\left(\sum_{i_{A_{1}}} \sum_{i_{A_{2}}} \frac{q_{m, 1} q_{m, 2}}{\left|r_{1^{+} 2^{+}}\right|^{2}}\right. \\
& +\sum_{i_{A_{1}}} \sum_{i_{A_{2}}} \frac{\left(-q_{m, 1}\right) q_{m, 2}}{\left|r_{1^{-} 2^{+}}\right|^{2}} \\
& +\sum_{i_{A_{1}}} \sum_{i_{A_{2}}} \frac{q_{m, 1}\left(-q_{m, 2}\right)}{\left|r_{1^{+} 2^{-}}\right|^{2}} \\
& \left.+\sum_{i_{A_{1}}} \sum_{i_{A_{2}}} \frac{\left(-q_{m, 1}\right)\left(-q_{m, 2}\right)}{\left|r_{1^{-} 2^{-}}\right|^{2}}\right) .
\end{aligned}
$$

For each pole face, the summation is achieved by adding over the cylindrical coordinate space (i.e., $r$ and $\phi$ dependence, as will be indicated by the indexes $i_{r}$ and $\left.i_{\phi}\right)$. For the cylindrical coordinate space, the summation is in the general form of (6), where $N_{r}$ corresponds to the number of radial facet elements and $N_{\phi}$ is the number of azimuthal facet elements. In this study, the $\phi$ dependence is eliminated assuming that the stent's magnetic ring attachment and hand-held magnet both lie in the $\{r, \phi\}$ plain of a cylindrical coordinate system $\{r, \phi, z\}$ and are centered at $r=0$, but separated along the $z$-axis

$$
\sum_{i_{A_{1}}}=\sum_{i_{r, 1}=1}^{N_{r, 1}} \sum_{i_{\phi, 1}=1}^{N_{\phi, 1}}=2 \pi \sum_{i_{r, 1}=1}^{N_{r, 1}} .
$$

The full summations are then given for the cylindrical coordinate system in (7), where + denotes the north pole face, - denotes the south pole face, index 1 indicates the hand-held magnet, and index 2 indicates the magnetic stent attachment

$$
\begin{aligned}
F_{m}= & \frac{1}{4 \pi \mu_{0}}\left(2 \pi \sum_{i_{r, 1}=1}^{N_{r, 1}}\left(2 \pi \sum_{i_{r, 2}=1}^{N_{r, 2}} \frac{q_{m, 1^{+}} q_{m, 2^{+}}}{\left|r_{1^{+} 2^{+}}\right|^{2}}\right)\right. \\
& +2 \pi \sum_{i_{r, 1}=1}^{N_{r, 2}}\left(2 \pi \sum_{i_{r, 2}=1}^{N_{r, 2}} \frac{\left(-q_{m, 1^{-}}\right) q_{m, 2^{+}}}{\left|r_{1^{-} 2^{+}}\right|^{2}}\right)
\end{aligned}
$$




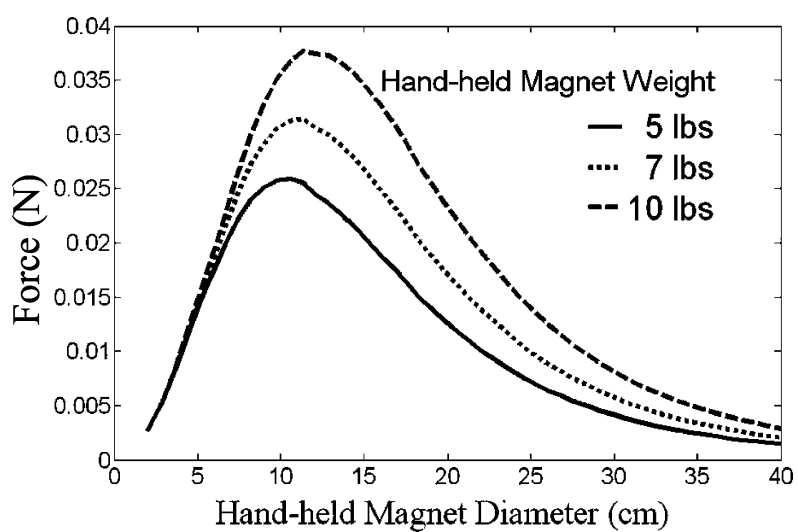

Fig. 3. Force at $10 \mathrm{~cm}$ separation between the hand-held magnet and the magnetic ring. Three different hand-held magnet weights are shown: 5, 7, and $10 \mathrm{lbs}$. In each case, there is an optimal diameter (with corresponding length governed by the weight) that maximizes the compression force, evaluated at $10 \mathrm{~cm}$ from a magnetic ring with dimensions of $\mathrm{OD}=1 / 8^{\prime \prime}, \mathrm{ID}=1 / 16^{\prime \prime}$, and length $=1 / 4^{\prime \prime}$, also of N42-grade NdFeB material.

$$
\begin{aligned}
& +2 \pi \sum_{i_{r, 1}=1}^{N_{r, 1}}\left(2 \pi \sum_{i_{r, 2}=1}^{N_{r, 2}} \frac{q_{m, 1^{+}}\left(-q_{m, 2^{-}}\right)}{\left|r_{1^{+} 2^{-}}\right|^{2}}\right) \\
& \left.+2 \pi \sum_{i_{r, 1}=1}^{N_{r, 1}}\left(2 \pi \sum_{i_{r, 2}=1}^{N_{r, 2}} \frac{\left(-q_{m, 1^{-}}\right)\left(-q_{m, 2^{-}}\right)}{\left|r_{1^{-} 2^{-}}\right|^{2}}\right)\right) .
\end{aligned}
$$

For the hand-held magnet, the radial facet segment length was set to $1.25 \mathrm{~mm}$, chosen as a tradeoff between time to run the simulation $(<5 \mathrm{~min})$ while remaining significantly larger than the shortest separation distance considered in simulation $(>1 \mathrm{~cm})$. Therefore, each iteration of $i_{r, 1}$ corresponds to an $1.25 \mathrm{~mm}$ increment along the radial axis. Therefore, the total number of radial facets changes with hand-held magnet diameter (e.g., $N_{r, 1}=30$ for a $3.81 \mathrm{~cm}=3^{\prime \prime}$ magnet diameter). For the magnetic ring of the stent, the radial facet segment length was set to $40 \mu \mathrm{m}$ and $N_{r, 2}=20$ throughout, since only the ring's length, and not its radii, change. In the case of the ring magnet, $i_{r, 2}$ was iterated such that $i_{r, 2}=1$ corresponded to the inner radius of the ring (i.e., $1 / 16^{\prime \prime}$ ), and $i_{r, 2}=N_{r, 2}$ corresponded to the ring's outer radius (i.e., $1 / 8^{\prime \prime}$ ).

This numerical model was then used to simulate 1) the force between the two magnets as a function of hand-held magnet diameter, as shown in Fig. 3 for various hand-held magnet weights; and 2) the force between the two magnets as a function of separation, as shown in Fig. 4, which was compared with experimental ex vivo results. The magnetic force on axially magnetized $\mathrm{N}-42$ grade $\mathrm{NdFeB}$ rings of $1.5875 \mathrm{~mm}\left(1 / 16^{\prime \prime}\right) \mathrm{ID}$, and $3.175\left(1 / 8^{\prime \prime}\right)$ and $6.35 \mathrm{~mm}\left(1 / 4^{\prime \prime}\right)$ length due to a hand-held cylindrical magnet of increasing diameter and fixed weight was simulated by means of (7). For the hand-held magnet, N-42 grade NdFeB was also simulated and later used in experiment.

While the model assumed concentric and perpendicular alignment between the hand-held magnet and the magnetic stent, there is no guarantee that this will be the case in clinical testing. However, the retrieval system does not necessarily need to be entirely blind. For example, if upon placement of the stent, a

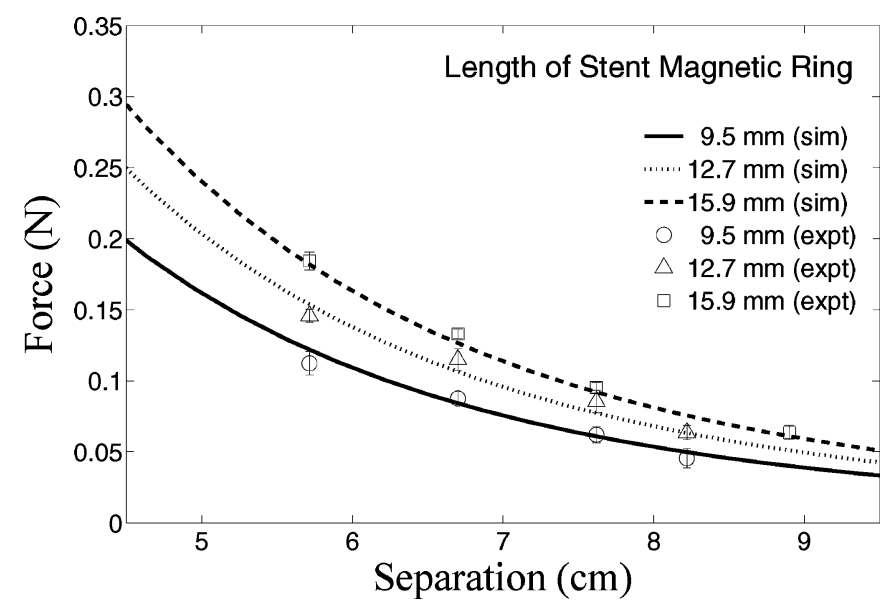

Fig. 4. Attractive forces between magnetic rings of various lengths (9.5, 12.7, and $15.9 \mathrm{~mm}$ ) as predicted by 1) simulation in MATLAB and 2) experimental investigation using the bending-beam force gauge. The hand-held magnet in each case is $\mathrm{N}-42$ grade $\mathrm{NdFeB}$ with a diameter of $3^{\prime \prime}$ and a length of $2^{\prime \prime}$, polarized along its length. There is good agreement between simulation and experiment over the range of separation considered.

fluoroscopic image of the patient is obtained (this is often obtained in any case for diagnostic purposes), a patch or similar marker could be attached to the patient's skin indicating the closest cutaneous surface perpendicular to the magnetic stent. When the patient returns some days later for removal of the stent, this marker could be assumed relatively concentric with the magnetic stent and the hand-held magnet placed roughly perpendicular, as idealized in the simulation.

The attractive force between the magnetic rings and handheld magnets of changing diameter and three different weights is shown in Fig. 3 for $10 \mathrm{~cm}$ separation between the magnetic ring and the hand-held magnet. For 2.268- (5 lbs), 3.175- (7 lbs), and $4.536-\mathrm{kg}$ (10 lbs) hand-held magnets, the maximum attractive force at $10 \mathrm{~cm}$ separation occurs at $10.53,11.30$, and $11.31 \mathrm{~cm}$ hand-held magnet diameters, respectively. The maximum simulated force associated with each optimized diameter is 25.87 , 31.4 , and $37.6 \mathrm{mN}$, respectively. The associated hand-held magnet length is determined by the weight constraint, where $\mathrm{NdFeB}$ has a density of $8.2 \mathrm{~g} / \mathrm{cm}^{3}$. To facilitate easy maneuvering of the hand-held magnet, a 5 lbs weight limit was arbitrarily imposed such that a diameter of $10.53 \mathrm{~cm}$ and corresponding length of $3.17 \mathrm{~cm}$ is found to be optimal.

The closest "off-the-shelf" N42 NdFeB cylinder was purchased (KJ Magnetics, Jamison, PA) with dimensions of 7.62 $\mathrm{cm}\left(3^{\prime \prime}\right)$ in diameter and $5.08 \mathrm{~cm}\left(2^{\prime \prime}\right)$ in length, and weighing approximately $1.74 \mathrm{~kg}$ or $3.84 \mathrm{lbs}$. The magnet is coated with a $50 \mu \mathrm{m} \mathrm{Ni-Cu}-\mathrm{Ni}$ layer for oxidation protection. This was the hand-held magnet used in all subsequent experiments.

The attractive force between a cylindrical hand-held magnet and the same magnetic ring was evaluated, again with reference to (7), but by using the diameter of the purchased hand-held magnet $(7.62 \mathrm{~cm})$. The force as a function of separation between the distal face of the magnetic ring and nearest face of the handheld magnet is shown in Fig. 4, where the two magnets are coaxial and centered at $r=0$. Simulated results for the force 


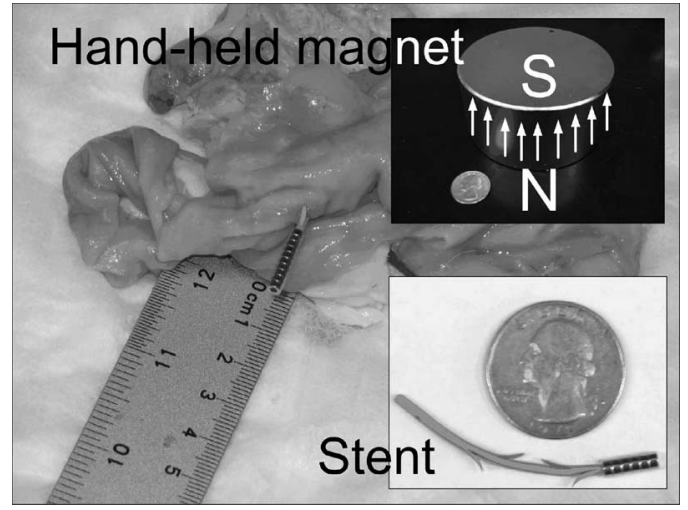

Fig. 5. Ex vivo test apparatus is shown. This consists of a large N-42 grade $\mathrm{NdFeB}$ parent magnet ( $3^{\prime \prime}$ diameter and $2^{\prime \prime}$ depth), a 5-3 or 5-5 pancreatic stent and a magnetic ring attachment consisting of a long $\mathrm{N}-42$ grade $\mathrm{NdFeB}$ rare earth magnet ( $1 / 8^{\prime \prime} \mathrm{OD}, 1 / 16^{\prime \prime} \mathrm{ID}$, and various lengths).

were obtained for three different lengths of magnetic rings: $6.35\left(1 / 4^{\prime \prime}\right), 9.525\left(3 / 8^{\prime \prime}\right)$, and $12.7 \mathrm{~mm}\left(1 / 2^{\prime \prime}\right)$. In each case, the ring's ID is $1.5875 \mathrm{~mm}\left(1 / 16^{\prime \prime}\right)$ and the OD is $3.175 \mathrm{~mm}$ $\left(1 / 8^{\prime \prime}\right)$.

\section{B. Ex Vivo Testing}

The force was then evaluated experimentally for each of the three ring lengths as a function of separation from the hand-held magnet. This was achieved by suspending the magnetic ring from a simply supported thin aluminum beam ( $72^{\prime \prime}$ long, $1 / 2^{\prime \prime}$ wide, and 1/16" thick, F.D. and Sons Hardware, Chicago, IL). An initial calibration of the beam deflection, $s$ versus point load $p$, at the beam's center was undertaken, and using a least-squares fitting algorithm, the calibration yielded an analytical expression for the beam deflection as a function of known load $s(p)$. The inverse relation [i.e., $p(s)$ ] was used to measure the force of attraction between the hand-held magnet (at a distance $s$ ) and the magnetic ring's closest face using the three different ring lengths already simulated. The results for mean and standard deviation of the force are shown in Fig. 4, where the error bars corresponding to the standard deviation were found from five iterations of each experiment.

Close agreement was observed between the simulated and experimental data while the beam deflection remained a linear function of loading.

Capture distance using the purchased hand-held magnet was measured in the ex-vivo porcine ampulla, as indicated in Fig. 5. Magnetic rings were attached to standard pancreatic stents using medical-grade adhesive (Loctite 4541 PrismGel, Henkel Corp., Düsseldorf, Germany). Three different magnetic rings were attached to GPSO-5-3 and GPSO-5-5 Geenan pancreatic stents (Cook Medical, Bloomington, IN) (9.5 (3/8" $)$, $12.7\left(1 / 2^{\prime \prime}\right)$, and $15.9 \mathrm{~mm}\left(5 / 8^{\prime \prime}\right)$ long $)$ as indicated in Table I and shown in Fig. 5 with the GPSO-5-3 stent. The effect of the barbs on capture distance was also investigated. In each case, the magnetic ring overlapped with $1 / 16^{\prime \prime}$ of the stent's plastic length, as indicated in Fig. 5; therefore, the overall extension to the stent's original plastic length was slightly less than that of the attached
TABLE I

Ex Vivo Capture Distances With VARying Magnetic Ring Length

\begin{tabular}{r|l|r|l} 
Length $(\mathrm{cm})$ & Distance $(\mathrm{cm})$ & Force $(\mathrm{mN})$ & Barbs (Y/N) \\
\hline 9.5 & $2.5 \pm 0.1$ & 680 & $\mathrm{Y}$ \\
9.5 & $9.0 \pm 0.3$ & 59.2 & $\mathrm{~N}$ \\
9.5 & $9.0 \pm 0.6^{*}$ & 59.2 & $\mathrm{~N}$ \\
12.7 & $4.0 \pm 0.1$ & 361.1 & $\mathrm{Y}$ \\
12.7 & $10.0 \pm 0.4$ & 44.1 & $\mathrm{~N}$ \\
12.7 & $9.0 \pm 0.4^{*}$ & 59.2 & $\mathrm{~N}$ \\
15.9 & $4.0 \pm 0.1$ & 361.1 & $\mathrm{Y}$ \\
15.9 & $10.0 \pm 0.3$ & 44.1 & $\mathrm{~N}$
\end{tabular}

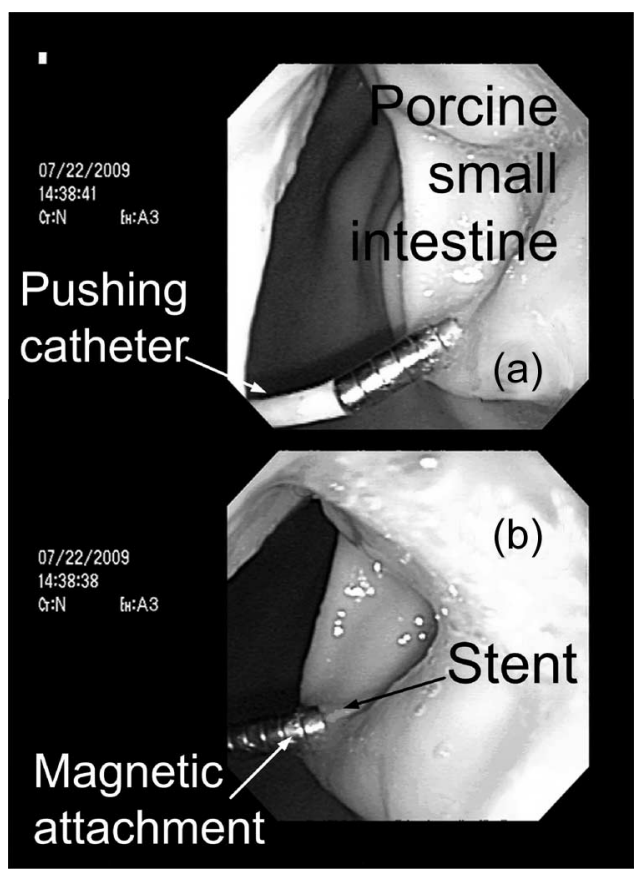

Fig. 6. In vivo introduction of the modified stent is shown during insertion by means of an endoscopic pushing catheter, shown in (a), at the hepatopancreatic ampulla in the porcine cadaver. Pancreatic stent is inserted complete with magnetic attachment, shown in (b). The stent was subsequently retrieved from the ampulla using the external, hand-held magnet.

magnetic ring. The stent was then manually inserted into the porcine ampulla such that the magnetic attachment extruded from the insertion point (see Fig. 5). Then, the hand-held magnet approached the stent such that the stent was approximately centered with the hand-held magnet's center along the line of approach. Capture was achieved once the stent had completely left the duct having been pulled into the hand-held magnet. Mean capture distance is recorded over five iterations in Table I for each of the magnetic rings attached, where the asterisk * indicates the use of 5-5 rather than 5-3 pancreatic stents.

\section{In Vivo Testing}

Subsequent endoscopic deployment of the stent, complete with a $9.5-\mathrm{mm}$ magnetic ring attached, was demonstrated in the live porcine model, as shown in Fig. 6. A 38.7-kg Yorkshire male pig was the subject of a standard ERCP procedure. A protective overtube was inserted into the esophageal tract and a standard 
gastric endoscope (Olympus Optical Co. Ltd, Tokyo, Japan) advanced through the stomach into the first portion of the small intestine. A Hydra Jagwire (Boston Scientific Corp., Natick, MA) was placed deep into the biliary tree following endoscopic localization of the ampulla. This guidewire served as the tramline for the endoscopic introduction of the magnetic stent. A magnetic stent comprising a modified 5-3 Geenan pancreatic stent with a 9.5-mm-long magnetic ring attached was back loaded through the instrument channel of a standard gastric endoscope such that the guidewire ran through the stent's lumen. The endoscope was reintroduced in vivo along the guidewire, and a customized stent introducer (Cook Medical, Bloomington, IN) was used to push the magnetic stent into position in vivo. After placement, the magnetic portion of the stent still extended from the ampulla. The guidewire was removed while the pusher remained in place, maintaining the stent's position within the biliary tree. After removal of the guidewire, the endoscope was slightly withdrawn so as to maintain line of sight with the stent at the ampulla. The hand-held magnet was then introduced external to the animal and, after some cutaneous massage, was able to withdraw the magnetic stent from the biliary tree into the small intestine. Magnetic retrieval was achieved at an estimated separation of 5-6 cm and the retrieval was captured on endoscopic video.

\section{DISCUSSION}

The overriding goal of this study was to evaluate the feasibility of a magnetic stent retrieval system using an external hand-held magnet to remove stents from the pancreaticobiliary tree. In particular, the study sought 1) to propose optimal geometries for the hand-held and stent magnets; 2) to evaluate the required attractive force by means of numerical simulation and experimental investigation; and 3) to test the design in both ex vivo and in vivo porcine models. Due to differences in anatomy, the distance in capture distances for human versus porcine in vivo experiments could be up to $5 \mathrm{~cm}$, where the distance will increase in human subjects.

A target capture distance of $10 \mathrm{~cm}$ between the external handheld magnet and the stent magnet was identified from review of fluoroscopic imagery in human patients. For the stent magnet, the ID and OD were constrained by the plastic stent diameter and the instrument channel of the standard gastroscope, respectively. Therefore, magnetic rings with ID of $1.5875 \mathrm{~mm}\left(1 / 16^{\prime \prime}\right)$ and OD of $3.175 \mathrm{~mm}\left(1 / 8^{\prime \prime}\right)$ were used throughout. Various magnetic ring lengths were investigated $(9.5,12.7$, and $15.9 \mathrm{~mm})$. The optimized hand-held magnet was a cylindrical permanent magnet of $11.3 \mathrm{~cm}$ diameter, $4.3 \mathrm{~cm}$ long, weighing $7 \mathrm{lbs}$ with a maximum simulated force of $31.4 \mathrm{mN}$ due to magnetic attraction at $10 \mathrm{~cm}$ for the $12.7-\mathrm{mm}$ ring magnet. The experimental performance of the closest commercially available cylindrical magnet was then evaluated. It had dimensions of $7.62 \mathrm{~cm}$ in diameter and $5.08 \mathrm{~cm}$ in length, weighed $3.84 \mathrm{lbs}$, and consisted of N-42 grade, axially magnetized NdFeB. The ring magnets attached to the plastic stents were of similar magnetic material. The performance of this hand-held magnet in retrieval of the magnetic stent in the ex-vivo porcine model, as given in Table I, indicate that capture distance is dependent on 1) the length of the magnetic ring attached to the stent and 2) the presence of barbs on the stent, but does not vary significantly with the length of the plastic portion of the stent. While the ex vivo experiment cannot accurately represent the in vivo compressive forces acting on the stent within the biliary tree, which arise due the surrounding anatomy in vivo, it should also be considered that the lubrication of the biliary tree associated with the ex vivo experiments is expected to be significantly reduced compared to that in the live animal. This hypothesis was subsequently validated, at least in animal studies, by successful retrieval at an estimated separation of 5-6 cm in the in vivo porcine model. Human trials will be needed to test this hypothesis in vivo.

Extensive ex vivo tests have shown that the absence of barbs is critical to successful capture with the hand-held magnet, where capture distance increased from $2.5 \pm 0.1 \mathrm{~cm}$ with stent barbs intact to $9.0 \pm 0.3 \mathrm{~cm}$ after their removal for the $9.5 \mathrm{~mm}$ magnetic ring. The corresponding decrease in the required magnetic attractive force at capture is over an order of magnitude (680 $\mathrm{mN}$ versus $59.2 \mathrm{mN}$ ), where the decrease in resistance is due to the elimination of the stent barbs. Similar results were obtained independent of plastic length and magnetic ring length. The primary finding is that a smooth, barbless stent is optimal for capture with a hand-held magnet at the target distance of 10 $\mathrm{cm}$.

The second finding of the ex vivo testing is the increase in capture distance achieved by increasing the length of the magnetic ring attachment. In the absence of barbs, the addition of an extra $3.175 \mathrm{~mm}\left(1 / 8^{\prime \prime}\right)$ length of magnetic material to the original $6.35 \mathrm{~mm}\left(1 / 4^{\prime \prime}\right)$ length resulted in an increase of $\sim 9 \%$ in capture distance $(10 \mathrm{~cm}$ versus $9 \mathrm{~cm})$, and an associated reduction in the required magnetic attractive force $(44.1 \mathrm{mN}$ versus 59.2 $\mathrm{mN})$. However, subsequent increases in magnetic ring length to $15.9 \mathrm{~mm}$ caused no increase in capture distance. It is thought that as the ring attached to the stent is increased, the face closest to the hand-held magnet becomes increasingly dominant in determining capture. While longer magnetic rings result in a larger capture distance, they are expected to have two undesired results in subsequent survival studies: 1) the stent is heavier and, therefore, more likely to fall into the small-bowel prematurely and 2) there is increased risk of occlusion or blockage of the small intestine due to the magnetic body in the lumen. For these reasons, rings not exceeding $12.7 \mathrm{~mm}$ length will be used in future survival studies. Increases in the length of the plastic portion of the stent resulted in no change (for the 9.5-mm-long ring) or little ( $\sim 10 \%$ for the $12.7-\mathrm{mm}$ ring attached) decrease in capture distance. This is most likely because tissue friction in the pancreaticobiliary tree is most pronounced at the ampulla from where the stent protrudes and friction is not as significant further up the duct.

The endoscopic deployment of the magnetic stent was largely uneventful. Total procedure time was on the order of 30 minutes where this was largely spent on localization of the ampulla by endoscopic visualization. This step is frequently time consuming in ERCP procedures. However, subsequent deployment, withdrawal of the endoscope and retrieval of the magnetic stent were surprisingly straightforward. Clearly, the estimated separation distance of 5-6 cm upon retrieval was less than that expected 
in the human anaotomy. However, the successful demonstration in the porcine model supplies evidence that the procedure may indeed obviate the need for a second endoscopic procedure to remove pancreatic and biliary stents in vivo.

\section{CONCLUSION}

The goal of this study was to design an optimized magnetic retrieval mechanism for pancreatic and biliary stents with a magnetic attachment, and to test the resulting design in the ex vivo porcine model. Successful retrieval of the magnetic stent at a target distance of $10 \mathrm{~cm}$ from the external hand-held magnet requires a smooth plastic stent with no barbs. In this case, a magnetic attractive force of approximately $69 \mathrm{mN}$ was sufficient to overcome the tissue friction associated with capture from the pancreaticobiliary duct using a $12.7-\mathrm{mm}$-long magnetic stent ring and the hand-held magnet in this study. This force decreased to $41 \mathrm{mN}$ when the magnetic stent ring was extended to 15.9 $\mathrm{mm}$ in length.

While the results of this preliminary analysis and experimentation are encouraging, it remains to be seen how the system will perform in in vivo porcine survival trials, which will begin in 2010 and subsequent human pilot studies. The primary questions to be answered by these studies are 1) whether plastic stents without barbs will remain in place over the time periods of interest (typically 3-days) and 2) if tissue friction in the human model will differ significantly from the ex vivo porcine model, thus, resulting in more or less difficulty in stent retrieval. Smooth stents are not unprecedented in the clinical setting, where physicians routinely remove barbs for accelerated stent extrusion from the pancreaticobiliary tree. However, it remains to be seen how barbless stents and the addition of the magnetic attachment will affect premature extrusion in the porcine model. Pending these important investigations, the current results point to a promising and cost-effective method that has potential use in stent retrieval within the pancreaticobiliary tree.

\section{REFERENCES}

[1] E. Roeland and C. F. von Gunten, "Current concepts in malignant bowel obstruction management," Curr. Oncol. Rep., vol. 4, pp. 298-303, Jul. 2009.

[2] D. F. Hutcheon, "The role of endoluminal stents in gastrointestinal diseases," Adv. Surg., vol. 38, pp. 183-196, 2004.

[3] P. Singh, A. Das, G. Isenberg, R. C. K. Wong, M. V. Sivak, Jr., D. Agrawal, and A. Chak, "Does prophylactic pancreatic stent placement reduce the risk of post-ERCP acute pancreatitis? A meta-analysis of controlled trials," Gastrointest. Endosc., vol. 60, pp. 544-550, Oct. 2004.

[4] P. H. P. Davids, A. K. Groen, E. A. J. Rauws, G. N. J. Tytgat, and K. Huibregtse, "Randomised trial of self-expanding metal stents versus polyethylene stents for distal malignant biliary obstruction," Lancet, vol. 340, pp. 1488-1492, 1992.

[5] M. Equen, G. Roach, R. Brown, and T. Bennett, "Magnetic removal of foreign bodies from the esophagus, stomach, and duodenum," Arch. Otolaryngol._Head Neck Surg., vol. 66, pp. 698-706, Dec. 1957.

[6] E. Paulson and R. Jaffe, "Metallic foreign bodies in the stomach: Fluoroscopic removal with a magnetic orogastric tube," Radiology, vol. 174, pp. 191-194, Jan. 1990.

[7] D. Diehl, D. Adler, J. Conway, F. Farraye, S. Kantsevoy, V. Kaul, S. Kethu, R. Kwon, P. Marnula, S. Rodriguez, and W. Tierney, "Endoscopic retrieval devices," Gastrointest. Endosc., vol. 69, pp. 997-1003, May. 2009.

[8] H. Eggebrecht, H. Kuhl, G. M. Kaiser, S. Aker, M. O. Zenge, F. Stock, F. Breuckmann, F. Grabellus, M. E. Ladd, R. H. Mehta, R. Erbel, and H. H. Quick, "Feasibility of real-time magnetic resonance-guided stent-graft placement in a swine model of descending aortic dissection," Eur. Heart J., vol. 27, pp. 613-620, Mar. 2006.

[9] L. Feng, C. Dumoulin, S. Dashnaw, R. Darrow, R. de la Paz, P. Bishop, and J. Pile-Spellman, "Feasibility of stent placement in carotid arteries with real-time MR imaging guidance in pigs," Radiology, vol. 234, pp. 551$557,2005$.

[10] S. Kos, R. Huegli, E. Hofmann, H. Quick, H. Kuehl, S. Aker, G. Kaiser, P. Borm, A. Jacob, and D. Bilecen, "First magnetic resonance imaging-guided aortic stenting and cava filter placement using a polyetheretherketone-based magnetic resonance imaging-compatible guidewire in swine: Proof of concept," CardioVasc. Intervent. Radiol., vol. 32, pp. 514-521, May 2009.

[11] E. Spuentrup, A. Ruebben, T. Schaeffter, W. J. Manning, R. W. Gunther, and A. Buecker, "Magnetic resonance-guided coronary artery stent placement in a swine model," Circulation, vol. 105, pp. 874-879, Feb. 2002.

[12] H. H. Quick, M. E. Ladd, D. Nanz, K. P. Mikolajczyk, and J. F. Debatin, "Vascular stents as RF antennas for intravascular MR guidance and imaging," Magn. Reson. Med., vol. 42, pp. 738-745, 1999.

[13] S. Gabriel, R. Ackermann, and M. Castresana, "A new technique for placement of nasoenteral feeding tubes using external magnetic guidance," Crit. Care Med., vol. 25, pp. 641-645, 1997.

[14] S. Gabriel, B. McDaniel, D. W. Ashley, M. L. Dalton, and T. C. Gamblin, "Magnetically guided nasoenteral feeding tubes: A new technique," Amer. Surg., vol. 67, pp. 544-548, Jun. 2001.

[15] H. Chen, A. D. Ebner, M. D. Kaminski, A. J. Rosengart, and J. A. Ritter, "Analysis of magnetic drug carrier particle capture by a magnetizable intravascular stent-2: Parametric study with multi-wire two-dimensional model," J. Magn. Magn. Mater, vol. 293, pp. 616-632, May 2005.

[16] B. Gleich, N. Hellwig, H. Bridell, R. Jurgons, C. Seliger, C. Alexiou, B. Wolf, and T. Weyh, "Design and evaluation of magnetic fields for nanoparticle drug targeting in cancer," IEEE Trans. Nanotechnol., vol. 6, no. 2, pp. 164-170, Mar. 2007.

[17] M. O. Avilés, H. Chen, A. D. Ebner, A. J. Rosengart, M. D. Kaminski, and J. A. Ritter, "In vitro study of ferromagnetic stents for implant assistedmagnetic drug targeting," J. Magn. Magn. Mater., vol. 311, pp. 306-311, Apr. 2007.

[18] A. J. Rosengart, M. D. Kaminski, H. Chen, P. L. Caviness, A. D. Ebner, and J. A. Ritter, "Magnetizable implants and functionalized magnetic carriers: A novel approach for noninvasive yet targeted drug delivery," J. Magn. Magn. Mater, vol. 293, pp. 633-638, May 2005.

[19] B. B. Yellen, Z. G. Forbes, D. S. Halverson, G. Fridman, K. A. Barbee, M. Chorny, R. Levy, and G. Friedman, "Targeted drug delivery to magnetic implants for therapeutic applications," J. Magn. Magn. Mater, vol. 293, pp. 647-654, May 2005.

[20] Z. Forbes, B. Yellen, D. Halverson, G. Fridman, K. Barbee, and G. Friedman, "Validation of high gradient magnetic field based drug delivery to magnetizable implants under flow," IEEE Trans. Biomed. Eng., vol. 55, no. 2, pp. 643-649, Feb. 2008.

[21] S. Tamaz, R. Gourdeau, A. Chanu, J. Mathieu, and S. Martel, "Realtime MRI-based control of a ferromagnetic core for endovascular navigation," IEEE Trans. Biomed. Eng., vol. 55, no. 7, pp. 1854-1863, Jul. 2008.

[22] A. Chanu and S. Martel, "Real-time software platform design for in-vivo navigation of a small ferromagnetic device in a swine carotid artery using a magnetic resonance imaging system," in Proc. 29th Annu. Int. Conf. IEEE Eng. Med. Biol. Soc. (EMBS), 2007, pp. 6584-6587.

[23] E. P. Furlani, Permanent Magnet and Electromechanical Devices: Materials, Analysis, and Applications. San Diego, CA: Academic, 2001.

Pádraig Cantillon-Murphy (M'03) received the B.E. degree in Electrical and Electronic Engineering from the University College Cork, Ireland, in 2003, and the M.S. and Ph.D. degrees from the Department of Electrical Engineering and Computer Science, Massachusetts Institute of Technology (MIT), Cambridge, in 2005 and 2008, respectively. His doctoral thesis examined the confluence of magnetic resonance imaging and magnetic nanoparticle dynamics. Since 2008, his postdoctoral research has been concerned with the role of magnetics in minimally invasive procedures, in collaboration with Brigham and Women's Hospital, Boston.

He is currently a Postdoctoral Research Fellow in the Research Laboratory of Electronics, MIT, Cambridge, and also a Research Fellow at Brigham and Women's Hospital, Boston, MA. 
Marvin Ryou received the B.A. degree from the University of California, Berkeley, and the M.D. degree from Harvard Medical School, Boston, MA, in 1998 and 2003, respectively.

$\mathrm{He}$ was an Internist, Hospitalist, and Staff Researcher at Brigham and Women's Hospital, Boston, MA. He is currently a Clinical Fellow in the Gastroenterology Division of Brigham and Women's Hospital and Harvard Medical School. He will begin an advanced fellowship in therapeutic endoscopy at Brigham and Women's Hospital in July 2010. His research interests include bariatric endoscopy, natural orifice transluminal endoscopic surgery (NOTES) and the role of magnetics in endoscopic therapy.

Sohail N. Shaikh received the M.D. degree from the Universidad Techologica de Santiago, Dominican Republic, in 1999, and Residency in Internal Medicine at St. Michael's Medical Center, Newark, NJ, in 2006.

He was a Research Fellow in Gastroenterology at St. Joseph's Regional Medical Center, Paterson, NJ, from 2008-2009, and Brigham and Women's Hospital, Boston, MA, in 2009. His research interests include novel instrumentation for therapeutic endoscopy and natural orifice transluminal endoscopic surgery (NOTES). He is an Advanced Endoscopy Fellow and Clinical Instructor at the University of Arizona Medical Center, Tucson.

Dan Azagury received the M.D. degree from Geneva University Medical School, Geneva, Switzerland, in 2001.

He completed general and gastroenterolgy surgical training in Switzerland and became a board-certified General Surgeon in 2008. He is currently a Research Fellow in Dr. Christopher Thompson's laboratory, Brigham and Women's Hospital, Boston, MA and Harvard Medical School, Boston, MA. His research interests include minimally invasive surgery, natural orifice and endoluminal surgery, bariatric surgery, nutrition in surgery, and device and procedure development. He will begin an Advanced Surgery Fellowship at Brigham and Women's Hospital in July 2010.

Michele Ryan graduated with a Bachelor's degree in biology (minors in chemistry, philosophy, and fine arts) from LeMoyne College, Syracuse, NY, in May 2003, and graduated from the Master's program at the Department of Public Health, University of Massachusetts, Amherst with a degree in biostatistics and epidemiology in June 2005.

She is the Research Laboratory Manager for Dr. Christopher C. Thompson in the Division of Gastroenterology, Hepatology, and Endoscopy at Brigham and Women's Hospital, Boston, MA. She has been worked with Dr. Thompson since October 2005 managing all aspects of his research including organization and execution of all clinical and non-clinical research projects, including manuscript preparation, grant writing, and protocol development. The research specifically focuses on Natural Orifice Transluminal Endoscopic Surgery (NOTES), endoscopic bariatric procedures (post-bariatric surgery and primary obesity), translational research and device development. She has coauthored three original manuscripts and over 15 abstracts to society meetings. She was also Assistant Editor for an issue of Techniques in Gastrointestinal Endoscopy with a topic of Techniques in Bariatric Endoscopy.
Christopher C. Thompson received the B.S. degree from the State University of New York, Binghamton, M.D. degree from Pennsylvania State University College of Medicine, Pennsylvania, and the M.S. degree from Pennsylvania State University, University Park, in 1992, 1996, and 2002, respectively.

$\mathrm{He}$ is currently the Director of Developmental Endoscopy at Brigham and Women's Hospital, Boston, MA, and an Associate Professor of Medicine at Harvard Medical School, Boston. He holds concurrent appointments at the Dana Farber Cancer Institute and Children's Hospital, Boston, and is the Codirector of the Center for Integration of Medicine and Innovative Technology (CIMIT) Working Group on Endoscopic Surgery. He has established an active animal laboratory geared toward device development and industry partnering. The laboratory currently has several active protocols for Natural Orifice Transluminal Endoscopic Surgery (NOTES) and the development of endoluminal devices. His research interests include advanced endoscopy, which applies to postsurgical complications, bariatric endoscopy, reflux, and pancreatic disease, NOTES, Endoscopic Suturing, gastroesophageal reflux disease, advanced endoscopy research and device development.

Jeffrey H. Lang (F'98) received the B.S., M.S., and Ph.D. degrees from the Department of Electrical Engineering and Computer Science, Massachusetts Institute of Technology (MIT), Cambridge, in 1975, 1977, and 1980, respectively.

He joined the Faculty of MIT in 1980, where he is currently a Professor of electrical engineering and computer science. He was the Associate Director of the MIT Laboratory for Electromagnetic and Electronic Systems during 19912003. He was an Associate Editor of Sensors and Actuators during 1991-1994. His research interests include the analysis, design and control of electromechanical systems with an emphasis on rotating machinery, microelectromechanical systems (MEMS) sensors, actuators and energy converters, and flexible structures. He has authored more than 200 papers published in various international journals and conferences, coauthored the book Foundations of Analog and Digital Electronic Circuits published by M. Kaufman, and holds 12 patents in the areas of electromechanics, MEMS, power electronics, and applied control.

Dr. Lang received four Best Paper Prizes from IEEE societies. He was a Fellow of Hertz Foundation. 